\title{
Asociación entre actividad física y salud mental positiva en estudiantes de medicina en México: un estudio transversal
}

\section{Association between physical activity and positive mental health in medical students in Mexico: a cross-sectional study}

\section{Associação entre atividade física e saúde mental positiva em estudantes de medicina do México: um estudo transversal}

\author{
Fouilloux, C., Fouilloux-Morales, M., Tafoya, S.A., Petra-Micu, I. \\ Departamento de Psiquiatría y Salud Mental, Facultad de Medicina, Universidad Nacional Autónoma de \\ México
}

\section{RESUMEN}

Esta investigación tuvo como objetivo identificar la relación entre la práctica de actividad física y aspectos positivos de la salud mental de alumnos de medicina en México. Se trata de un estudio transversal realizado con una muestra aleatoria de 190 estudiantes, con edad media de $19.0 \pm 1.4$ años, del primero y segundo grado de una escuela de medicina en la Ciudad de México. Se aplicó una batería de pruebas de salud mental positiva que comprendía escalas para evaluar la satisfacción de los jóvenes con la vida, su resiliencia, autoconcepto y compromiso, así como el IPAQ que evalúa su práctica de actividad física. Se llevaron a cabo análisis descriptivos, comparativos y de regresión lineal múltiple. Se encontró una asociación significativa del nivel de actividad física practicada por los alumnos con su satisfacción con la vida $(B=-1.64$, IC95\% $=-2.90,-0.38, p=.011)$ y su capacidad de resiliencia $(B=-2.41$, IC95\% $=-3.87,-0.95, p=.001)$, controlando por factores sociodemográficos. La actividad física tuvo una asociación significativa con aspectos positivos de la salud mental de estudiantes de medicina en México; por ello, intervenciones orientadas a favorecer la práctica de actividad física pueden diseñarse y utilizarse no sólo en el tratamiento y prevención de trastornos mentales, sino también para promover la salud mental entre esta población.

Palabras clave: Actividad física; satisfacción con la vida; resiliencia; autoconcepto; compromiso.

\section{ABSTRACT}

This study aimed to identify the relationship between the practice of physical activity and positive aspects of medical students' mental health in Mexico. This was a cross-sectional study that applied randomized sampling, and data from 190 students, with a mean age of $19.0 \pm 1.4$ years, from the first and second grades of a medical school in Mexico City were analyzed. The questionnaire comprised scales to assess students' satisfaction with life, resilience, selfconcept, and engagement, as well as the IPAQ that evaluates their practice of physical activity. Descriptive, comparative, and multiple linear regression analyses were carried out. A significant association was found between 


\section{Fouilloux, C., Fouilloux-Morales, M., Tafoya S.A., Petra-Micu, I.}

students' levels of physical activity, their satisfaction with life $(B=-1.64$, IC95\% $=-2.90,-0.38, p=.011)$ and their resilience skills $(B=-2.41$, IC95\% $=-3.87,-0.95, p=.001)$, controlling for sociodemographic factors. Physical activity had a significant association with positive aspects of Mexican medical students' mental health; thus, interventions aimed at improving physical activity practice can be designed and utilized not only in the treatment and prevention of mental disorders but also to promote mental health among this population.

Keywords: Physical activity; satisfaction with life; resilience; self-concept; engagement.

\section{RESUMO}

Este estudo teve como objetivo identificar a relação entre a prática de atividade física e aspectos positivos da saúde mental de estudantes de medicina do México. Este é um estudo transversal realizado com uma amostra aleatória de 190 alunos, com idade média de $19.0 \pm 1.4$ anos, do primeiro e segundo ano de uma escola de medicina na Cidade do México. Foi aplicado um questionário que incluía escalas para avaliar a satisfação dos alunos com a vida, sua resiliência, autoconceito e comprometimento, além do IPAQ que avalia sua prática de atividade física. Foram realizadas análises descritivas, comparativas e de regressão linear múltipla. Foi encontrada associação significativa entre os níveis de atividade física praticados pelos alunos, a satisfação com a vida $(B=-1.64$, IC95\% $=-2.90,-0.38$, $p=.011)$ e a resiliência $(B=-2.41$, IC95\% $=-3.87,-0.95, p=.001)$, controlando para fatores sociodemográficos. A atividade física teve associação significativa com aspectos positivos da saúde mental de estudantes de medicina mexicanos; portanto, intervenções destinadas a promover a prática de atividade física podem ser planejadas e utilizadas não apenas no tratamento e prevenção de transtornos mentais, mas também para promover a saúde mental dessa população.

Palavras chave: Atividade física; satisfação com a vida; resiliência; autoconceito; comprometimento.

\section{INTRODUCCIÓN}

La salud mental ha adquirido gradualmente mayor relevancia a nivel internacional y es que, al inicio del siglo XXI, más del $25 \%$ de la población en diferentes países había sufrido uno o más trastornos mentales o de la conducta a lo largo de su vida, ocasionando impacto en los individuos, las familias y las comunidades (Organización Mundial de la Salud [OMS], 2001). En México, una de cada cinco personas había sufrido al menos un trastorno mental en algún momento de su vida (Medina et al., 2003).

Existen varias opciones para el tratamiento de los trastornos mentales, que se sustentan en distintos enfoques teóricos. Entre las más estudiadas y utilizadas a nivel mundial, se encuentran el tratamiento farmacológico y el psicoterapéutico (Wegner, Helmich, Machado, Nardi, Arias-Carrión y Budde, 2014), que parten de una perspectiva tradicional donde se concibe a la salud mental como la ausencia de síntomas; bajo este modelo, la intervención se centra en la enfermedad mental y se termina por excluir de la atención a las personas que manifiestan una disminución de su nivel de salud mental, que no llega a constituir un cuadro de diagnóstico.
La forma de concebir a la salud ha evolucionado a lo largo de la historia, con base en el valor social y cultural que se le ha asignado en cada momento, así como en la metodología empleada para su análisis. Lo mismo sucede con el concepto de salud mental, y la manera en que se conciba servirá de punto de partida para estudiarla y abordarla. De este modo, así como desde la perspectiva tradicional mencionada con anterioridad, la intervención se centra en la enfermedad mental; a partir del modelo teórico de Jahoda (1958), en que la salud mental es concebida como la presencia de atributos individuales positivos, se aborda a ésta desde un enfoque orientado a la promoción de la salud en la población general y no sólo en los enfermos.

Este último modelo es un punto de referencia fundamental para los investigadores que consideran a la salud mental como algo más que la simple ausencia de enfermedad, así como para la OMS (2005), que la define como un estado de bienestar en el que el individuo está consciente de sus habilidades y es capaz de hacer frente al estrés normal de la vida, trabajar de forma productiva y contribuir al desarrollo de su comunidad. Bajo esta perspectiva, la práctica de actividad física se presenta como una alternativa para promover la salud mental. 


\section{Actividad física y salud mental en estudiantes de medicina mexicanos}

En términos generales, la actividad física se puede definir como "cualquier movimiento corporal producido por los músculos esqueléticos que resulta en gasto de energía" (Caspersen, Powell y Christenson, 1985). Si bien los términos ejercicio y actividad física se utilizan con frecuencia de manera indistinta, el primero es una subcategoría de la segunda y se refiere a una actividad física que ha sido planeada en forma estructurada con el propósito de mejorar o mantener uno o más componentes de la aptitud física (Caspersen et al., 1985; OMS, s.f.). La actividad física abarca el ejercicio, pero también otras actividades que entrañan movimiento corporal y se realizan como parte de la vida doméstica, laboral, escolar, recreativa y profesional de cada persona (López-Bárcena, González de Cossio-Ortiz y Rodríguez-Gutiérrez, 2006; OMS, s.f.).

Estudios en varios países sobre la asociación entre actividad física y salud mental han mostrado evidencia de los beneficios de la primera sobre la segunda; se ha observado, por ejemplo, que la actividad física previene o reduce el riesgo de desarrollar un trastorno psiquiátrico como ansiedad o depresión (AlmagroValverde, Dueñas-Guzmán y Tercedor-Sánchez, 2014; Borbón-Castro, Castro-Zamora, Cruz-Castruita, Banda-Sauceda, de la Cruz-Ortega, 2020; Dale, Vanderloo, Moore y Faulkner, 2019; Lebensohn et al., 2013; Rodríguez-Romo, Barriopedro, Alonso-Salazar y Garrido-Muñoz, 2015; Thome y Espelage, 2004; Wegner et al., 2014), niveles de estrés (Bass, Enochs y DiBrezzo, 2002; Lebensohn et al., 2013; LópezWalle, Tristán, Tomás, Gallegos-Guajardo, Góngora y Hernández-Pozo, 2020; Thome y Espelage, 2004), despersonalización (Lebensohn et al., 2013), así como agotamiento físico, cognitivo y emocional (Babenko y Mosewich, 2017).

Otras investigaciones también han revelado que la actividad física mejora aspectos relacionados con la salud mental, tales como: satisfacción con la vida (Fernández-Ozcorta, Almagro-Torres y Sáenz-LópezBuñuel, 2015; Lebensohn et al., 2013; Reigal, Hernández-Mendo, Juárez-Ruiz de Mier y MoralesSánchez, 2020; Thome y Espelage, 2004), resiliencia (Chacón-Cuberos, Puertas-Molero y Pérez-Cortés, 2017; Gómez-Chacón y Fernández-Martínez, 2020; Ozkara, Kalkavan, Alemdag y Alemdag, 2016; Romero-Barquero, 2014), autoconcepto (ÁlvarezRivera, Cuevas-Ferrera, Lara-Pot y González-
Hernández, 2015; Cachón-Zagalaz, Cuervo-Tuero, Zagalaz-Sánchez y González-González de Mesa, 2015; Dale et al., 2019; Kyle, Hernández-Mendo, Reigal-Garrido y Morales-Sánchez, 2016; MoralCampillo, Reigal-Garrido y Hernández-Mendo, 2020; Onetti-Onetti, Chinchilla-Minguet, Lourenço Martins y Castillo-Rodríguez, 2019), autoestima (Dale et al., 2019; Fernández-Ozcorta et al., 2015), autoeficacia (Reigal, Hernández-Mendo, et al., 2020; Reigal, Moral-Campillo, Morillo-Baro, Juárez-Ruiz de Mier, Hernández-Mendo y Morales-Sánchez, 2020; Reigal, Videra y Gil, 2014), compromiso (Gómez-Chacón y Fernández-Martínez, 2020), comprensión y regulación de los propios sentimientos y emociones (Fernández Ozcorta et al., 2015; Ligeza, Kałamała, Tarnawczyk, Maciejczyk y Wyczesany, 2019), bienestar emocional (Jamali et al., 2013; Lee, Spence, Tremblay y Carson, 2018; Muller, Dennis y Gorrow, 2006; Tamminen, Reinikainen, Appelqvist-Schmidlechner, Borodulin, Mäki-Opas y Solin, 2020; Terebessy, Czeglédi, Balla, Horváth y Balázs, 2016), motivación hacia el logro (Babenko y Mosewich, 2017; Babenko, Mosewich, Abraham y Lai, 2018) y funcionamiento social (Terebessy et al., 2016). Sin embargo, la práctica en exceso de actividad física puede resultar perjudicial tanto para la salud mental como física (NogueiraLópez, Salguero del Valle y Márquez-Rosa, 2017; Sánchez-Beleña y Alejo García-Naveira, 2017).

En el caso específico de los estudiantes de medicina en México, se ha observado que la carga de trabajo, el temor a obtener bajas calificaciones, así como la falta de tiempo para actividades recreativas, constituyen fuentes de estrés que pueden afectar su desempeño académico y sus relaciones interpersonales (GalvánMolina, Jiménez-Capdeville, Hernández-Mata y Arellano-Cano, 2017; Ortiz-León, ArechavaletaHernández, Tafoya-Ramos y Fouilloux-Morales, 2007). La consulta, medicación y canalización a terapia son las estrategias más socorridas para dar respuesta a esta problemática (Ortiz-León et al., 2007). Desafortunadamente, los servicios de consulta tienden a saturarse, además de implicar un costo institucional y personal. Se requieren entonces otras estrategias de intervención, en la que la actividad física se muestra como una opción adicional efectiva, no sólo para apoyar en el tratamiento de los trastornos mentales, sino como herramienta para promover el desarrollo de la salud mental. Mejorar el desarrollo de aspectos positivos de la salud mental tiene un impacto 


\section{Fouilloux, C., Fouilloux-Morales, M., Tafoya S.A., Petra-Micu, I.}

beneficioso en el rendimiento académico de los estudiantes (Peralta-Díaz, Ramírez-Giraldo y Castaño-Buitrago, 2006; Zhou et al., 2014). Además, la participación en programas de actividad física ha favorecido la adaptación de estudiantes de primer año de medicina a las altas exigencias de esta carrera, sin impactar negativamente en su productividad académica y convivencia (Ríos-Bustos, Torres-Vaca y de la Torre, 2017).

Pocos estudios se han enfocado en la asociación directa entre la práctica de actividad física y la salud mental positiva de estudiantes universitarios en la población mexicana. Es por tanto que esta investigación tuvo como objetivo identificar la relación entre la actividad física que practican alumnos de medicina en México y aspectos positivos de su salud mental, considerando algunas variables sociodemográficas de interés, así como el tiempo que les toma trasladarse del hogar a la escuela y de regreso. Con base en la literatura revisada, se espera encontrar una asociación positiva estadísticamente significativa entre la actividad física que practican los estudiantes de medicina y su satisfacción con la vida, resiliencia, autoconcepto y compromiso.

\section{MATERIAL Y MÉTODOS}

\section{Diseño de Investigación}

Investigación empírica de estrategia asociativa con un diseño predictivo transversal (Ato, López, \& Benavente, 2013).

\section{Participantes}

El estudio se realizó en estudiantes de primero y segundo año de la licenciatura de medicina de una universidad pública mexicana. Para su selección, se realizó un cálculo del tamaño de la muestra (MEDCALC, Versión 12.4.0) considerando un coeficiente de correlación de $.30, \alpha=.01, \mathrm{y} \beta=.10$, que resultó en un mínimo de 158 estudiantes. Dado que la universidad tiene grupos académicos previamente establecidos (alrededor de 32 grupos por año académico, con 35 a 40 alumnos en cada uno), se generó una tabla de números aleatorios con el propósito de seleccionar a tres grupos por cada año escolar (primero y segundo) y cubrir la cuota establecida. Estos grupos se conformaron por un total de 223 estudiantes. El currículo de la Facultad de Medicina de esta universidad incluye aspectos biomédicos y sociomédicos, en los dos primeros años; así como la práctica clínica en hospitales, durante los cinco semestres subsecuentes.

\section{Instrumentos}

Cédula de datos sociodemográficos. Se construyó un cuestionario ad hoc con el fin de recabar los datos de género, edad (años), año cursado (primero o segundo), tiempo de traslado casa-universidad-casa (minutos/días), nivel de escolaridad del padre y la madre [primaria, secundaria, bachillerato, carrera técnica, licenciatura, posgrado (especialidad, maestría, doctorado)], así como el nivel socioeconómico. Este último fue indagado con base en 10 preguntas a las que se les asignan puntuaciones, de cuya suma total (0366), se establecen seis niveles socioeconómicos (regla 10x6) de acuerdo con la Asociación Mexicana de Agencias de Inteligencia de Mercado y OpiniónAMAI (López-Romo, 2008). Del mismo modo, la escolaridad del padre y de la madre fue ponderada con base al impacto social y económico establecido por la AMAI, con valores que van de 0 a 72 .

Con la finalidad de evaluar los aspectos positivos de la salud mental, se utilizaron los instrumentos que aparecen a continuación, adaptados y validados de acuerdo con la muestra de estudio (con 486 alumnos universitarios mexicanos), con una escala tipo Likert de cinco puntos que va desde: $1=$ totalmente en desacuerdo a $5=$ totalmente de acuerdo.

Escala de satisfacción con la vida (Atienza, Pons, Balaguer y García-Merita, 2000). Versión en español de la escala de Diener, Emmons, Larsen y Griffin (1985), compuesta por cinco reactivos que evalúan el juicio global del individuo sobre su satisfacción con la vida, con reactivos como "En la mayoría de los aspectos, mi vida es como quiero que sea". Esta versión explicó $53.7 \%$ de la varianza, con cargas en sus reactivos de .63 a .83 (Atienza et al., 2000). La adaptación a estudiantes universitarios mexicanos obtuvo un alfa de Cronbach de .81 y una correlación de $r=-.50(p<.0001)$ con la versión en español de la Symptom Check List (SCL-90).

Escala breve de resiliencia. Traducción y adaptación al español de la escala de Smith et al. (2008), con seis reactivos como "Tiendo a recuperarme con rapidez, después de pasar por momentos difíciles", que evalúan la habilidad del individuo para recuperarse del estrés. La versión en inglés explicó $61 \%$ de la varianza, con 


\section{Actividad física y salud mental en estudiantes de medicina mexicanos}

cargas de .69 a .90 (Smith et al., 2008). La versión en español aplicada a estudiantes universitarios mexicanos obtuvo un alfa de Cronbach de .66 y una correlación con la SCL-90 de $r=-.40(p<.0001)$.

Escala de autoconcepto AC-2 (Morales, 2006). Compuesta por diez reactivos como "Me gustaría ser distinto(a)", que evalúan la percepción que el individuo tiene de sí mismo. En estudiantes universitarios españoles, esta versión explicó 70.3\% de la varianza (Morales, 2006). La adaptación aplicada a alumnos universitarios mexicanos obtuvo un alfa de Cronbach de .83 y una correlación de $r=-.63(p$ $<.0001)$ con la SCL-90.

Escala de compromiso. Versión en español de la escala de Schaufeli, Salanova, González y Bakker (2002), compuesta por 17 reactivos que evalúan en el individuo, su estado mental positivo y satisfactorio relacionado con el estudio, que se caracteriza por el vigor, la dedicación y la absorción durante las sesiones de estudio. Esta escala incluye reactivos como "Estoy entusiasmado(a) con mis estudios". Entre jóvenes universitarios españoles, la escala obtuvo un alfa de Cronbach de $.78, .84$ y .73 para las dimensiones de vigor, dedicación y absorción, respectivamente (Schaufeli et al., 2002). La versión adaptada a alumnos universitarios mexicanos obtuvo un alfa de Cronbach global de .88 y una correlación con la SCL-90 de $r=$ $-.34(p<.0001)$. Para el presente estudio, se consideró la puntuación total de la escala de compromiso para el análisis de los datos.

Para identificar los niveles de actividad física, se adaptó y validó el Cuestionario internacional de actividad física (versión corta). Versión en español del International Physical Activity Questionnaire (IPAQ, s.f.), que contiene siete reactivos a través de los cuales se puede obtener el tiempo (en minutos por semana) que el individuo dedica a caminar, permanecer sentado y realizar actividades moderadas o vigorosas. Los reactivos consideran preguntas como "Durante los últimos 7 días, ¿cuántos días realizaste actividades físicas vigorosas como levantar objetos pesados, excavar, ejercicios aeróbicos, correr, o pedalear rápido en bicicleta?" y "Usualmente, ¿cuánto tiempo en total realizaste esas actividades físicas vigorosas en los días que las realizaste?". Con esta información, se puede determinar si el nivel de actividad física reportada es baja, moderada o alta, con base en los criterios del manual del IPAQ. En este estudio, las categorías de nivel moderado y alto se unificaron en una sola categoría, de modo que únicamente dos grupos de nivel de práctica de actividad física fueron considerados para el análisis: bajo y moderado/alto.

Después de evaluar este cuestionario en doce países de los cinco continentes, su validez de criterio (contrastada con la actividad física registrada por un acelerómetro) obtuvo una correlación promedio de $r$ $=.30 \mathrm{y}$ un coeficiente de correlación promedio testretest (con ocho días entre una aplicación y otra) de $r$ $=.76$ (Craig et al., 2003). En la adaptación y validación realizada como parte de esta investigación con 88 jóvenes universitarios mexicanos, la validez convergente del IPAQ con el Cuestionario de actividad e inactividad de estudiantes mexicanos obtuvo una correlación de $r=.47(p<.01)$.

\section{Procedimiento}

Después de seleccionar a los grupos participantes, las investigadoras encargadas del estudio asistieron a las aulas e invitaron a los alumnos a participar, para lo cual se les leyó la información que explicaba la naturaleza de la investigación, así como el consentimiento informado; ambos incluidos mediante un texto breve al inicio del cuestionario aplicado, siguiendo las recomendaciones de Loue (2006). Quienes estuvieron de acuerdo en participar, respondieron en ese momento los cinco instrumentos de medición junto con la cédula de datos sociodemográficos. El proyecto fue aprobado por las comisiones de investigación y de ética de la Facultad de Medicina de la Universidad Nacional Autónoma de México de acuerdo con los principios de la Declaración de Helsinki y otras regulaciones a nivel nacional.

\section{Análisis estadístico}

Para todos los análisis, se utilizó el programa SPSS versión 23 (IBM Corp., 2014). Los niveles de actividad física se reportaron con frecuencias y porcentajes, mientras que las puntuaciones obtenidas de las escalas para evaluar los aspectos positivos de la salud mental se describieron a través de medias $(M)$ y desviaciones típicas $(D T)$.

Mediante la prueba de Kolmogórov-Smirnov se evaluó si los aspectos positivos de la salud mental (Satisfacción con la vida, Resiliencia, Autoconcepto y 


\section{Fouilloux, C., Fouilloux-Morales, M., Tafoya S.A., Petra-Micu, I.}

Compromiso) seguían una distribución normal, lo cual fue rechazado para todas las variables, por lo que se optó por utilizar estadística no paramétrica y un modelo lineal generalizado con un estimador robusto para el análisis multivariado (Fox, 2015).

Se realizaron correlaciones simples, mediante la prueba de Spearman, con el fin de establecer la asociación inicial entre las variables del estudio. La prueba U de Mann-Whitney se utilizó para comparar las puntuaciones de los aspectos positivos de la salud mental entre quienes practicaban actividad física a un nivel bajo y los que la realizaban a un nivel moderado o alto.

Finalmente, el modelo lineal generalizado de efectos principales con un estimador robusto se utilizó para investigar la asociación entre la práctica de actividad física de los estudiantes, las variables sociodemográficas (sexo, edad, grado escolar, nivel socioeconómico, escolaridad del padre y de la madre) y tiempo de traslado de la casa a la escuela y de regreso (todas éstas como variables independientes), con cada aspecto positivo de su salud mental (como variable dependiente). Para todos los análisis, el nivel de significancia se estableció en $p \leq .05$ con pruebas de hipótesis bilaterales.

\section{RESULTADOS}

De un total de 223 estudiantes, 8 rehusaron a colaborar en la investigación y 25 fueron excluidos debido a que no respondieron a todos los ítems presentes en los instrumentos de evaluación. De este modo, la muestra final quedó conformada por 190 alumnos, el $62.6 \%$ mujeres y el $37.4 \%$ hombres, el $54.2 \%$ se encontraban inscritos en primer año y el $45.8 \%$ en segundo, con una edad media de $19.0 \pm 1.4$ años.

Con respecto al nivel socioeconómico de los participantes el $42.1 \%$ reportaron el nivel socioeconómico más alto, mientras que el resto de los participantes (57.9\%) se distribuyó en los cuatro niveles siguientes; ninguno se ubicó en el nivel más bajo. En cuanto a la escolaridad de los padres, el $43.1 \%$ de las madres y el $52.7 \%$ de los padres habían cursado estudios universitarios; el resto había estudiado primaria, secundaria, una carrera técnica o comercial o el bachillerato. Finalmente, trasladarse de casa a la escuela y de regreso, les tomaba a los jóvenes una media de $126.0 \pm 74.3$ minutos por día.

Con base en los criterios establecidos en el manual del IPAQ, se encontró que 44 (23.2\%) de los estudiantes tenía un nivel de actividad física bajo, 87 (45.8\%) presentó un nivel moderado y $59(31.1 \%)$ un nivel alto, por lo que $68.9 \%$ de la población estudiada practicaba actividad física a un nivel moderado o alto. Por otra parte, todos los aspectos positivos de la salud mental evaluados en los alumnos obtuvieron puntuaciones superiores al valor medio correspondiente a cada escala. (Tabla 1).

Las correlaciones simples entre las variables de estudio se muestran en la Tabla 2, en la que se observa que el nivel de actividad física sólo se relacionó con la resiliencia.

Tabla 1

Medidas descriptivas, asimetría, curtosis y prueba de Kolmogorov-Smirnov para las medidas de aspectos positivos de la salud mental.

\begin{tabular}{lccccc}
\hline Aspectos positivos de la salud mental $^{\text {a }}$ & $\boldsymbol{M}$ & $\boldsymbol{D T}$ & $\boldsymbol{A}$ & $\boldsymbol{K}$ & $\boldsymbol{K}$ - $\boldsymbol{S}$ \\
\hline Satisfacción con la vida (5-25, valor medio 15 puntos) & 20.50 & 3.99 & -1.09 & 1.20 & $0.14^{* *}$ \\
Resiliencia (6-30, valor medio 18 puntos) & 20.39 & 4.55 & -0.13 & -0.02 & $0.07^{*}$ \\
Autoconcepto (10-50, valor medio 30 puntos) & 38.34 & 7.67 & -0.69 & -0.24 & $0.11^{* *}$ \\
Compromiso (17-85, valor medio 51 puntos) & 67.13 & 10.93 & -1.24 & 2.47 & $0.12^{* *}$ \\
\hline
\end{tabular}

Nota: $N=$ participantes, $M=$ media, $D T=$ desviación típica, $A=$ Asimetría, $K=$ Curtosis, $K-S=$ Estadístico de Kolmogórov-Smirnov.

${ }^{\text {a }}$ Los números entre paréntesis se refieren al rango de puntuaciones que cada escala de aspectos positivos de la salud mental puede generar e incluye su valor medio.

$* \mathrm{p}<.05, * * \mathrm{p}<.001$ 


\section{Actividad física y salud mental en estudiantes de medicina mexicanos}

Tabla 2

Correlaciones bivariadas entre las variables de estudio

\begin{tabular}{|c|c|c|c|c|c|c|c|c|c|}
\hline & 1 & 2 & 3 & 4 & 5 & 6 & 7 & 8 & 9 \\
\hline 1. Satisfacción con la vida & - & & & & & & & & \\
\hline 2. Resiliencia & $.43^{* *}$ & - & & & & & & & \\
\hline 3. Autoconcepto & $.66^{* *}$ & $.57^{* *}$ & - & & & & & & \\
\hline 4. Compromiso & $.63^{* *}$ & $.46^{* *}$ & $.55^{* *}$ & - & & & & & \\
\hline 5. Edad (años) & -.07 & -.07 & -.05 & .04 & - & & & & \\
\hline $\begin{array}{l}\text { 6. Tiempo de traslado casa-universidad-casa } \\
\text { (minutos/día) }\end{array}$ & $-.15^{*}$ & -.09 & -.02 & -.04 & $-.18^{*}$ & - & & & \\
\hline 7. Nivel socioeconómico (0 a 366 puntos) & $.18^{*}$ & .02 & .03 & -.02 & .08 & $-.36^{* *}$ & - & & \\
\hline 8. Escolaridad de la madre ( 0 a 72 puntos) & $.18^{*}$ & -.01 & -.01 & .01 & -.03 & $-.27^{* *}$ & $.50^{* *}$ & - & \\
\hline 9. Escolaridad del padre ( 0 a 72 puntos) & $.15^{*}$ & -.09 & -.06 & -.06 & .08 & $-.37^{* *}$ & $.64^{* *}$ & $.57^{* *}$ & - \\
\hline $\begin{array}{l}\text { 10. Nivel de actividad física practicado }(1= \\
\text { Bajo, } 2=\text { Moderado/Alto })\end{array}$ & .08 & $.15^{*}$ & .07 & -.04 & -.02 & -.03 & .01 & .06 & .08 \\
\hline
\end{tabular}

$* \mathrm{p}<.05, * *<.01$.

Al comparar las puntuaciones de las escalas de salud mental positiva entre el grupo de estudiantes que practicaba actividad física a un nivel moderado o alto y el que la realizaba a un nivel bajo, se encontraron diferencias significativas en satisfacción con la vida y resiliencia. Para la satisfacción con la vida, los alumnos del primer grupo obtuvieron puntuaciones medias mayores $(20.75 \pm 4.02)$ en comparación con los del segundo grupo (18.73 \pm 4.33$), Z=-1.98, p=.048$. Del mismo modo, para resiliencia, los alumnos con nivel moderado o alto obtuvieron puntuaciones medias mayores $(20.97 \pm 4.49)$ en comparación con los del nivel bajo (18.45 \pm 4.23$), Z=-3.11, p=.002$.

Tabla 3

Modelo para predecir satisfacción con la vida

\begin{tabular}{|c|c|c|c|c|c|}
\hline Variable/Categoría & $\boldsymbol{B}$ & $\boldsymbol{E} E$ & IC 95\% & $\chi^{2}$ Wald & $p$ \\
\hline (Intercepto) & 23.42 & 4.17 & {$[15.24,31.60]$} & 31.47 & .000 \\
\hline \multicolumn{6}{|l|}{ Nivel de actividad física practicado } \\
\hline $\begin{array}{l}\text { Moderado/Alto } \\
\text { Bajo }\end{array}$ & $\begin{array}{c}0 \\
-1.64\end{array}$ & 0.64 & {$[-2.90,-0.38]$} & 6.53 & .011 \\
\hline \multicolumn{6}{|l|}{ Sexo } \\
\hline $\begin{array}{l}\text { Mujer } \\
\text { Hombre }\end{array}$ & $\begin{array}{c}0 \\
-1.67\end{array}$ & 0.67 & {$[-2.97,-0.37]$} & 6.29 & .012 \\
\hline \multicolumn{6}{|l|}{ Grado de estudio } \\
\hline Segundo & 0 & & & & \\
\hline Primero & -0.53 & 0.62 & {$[-1.74,0.68]$} & 0.75 & .388 \\
\hline Edad (años) & -0.23 & 0.19 & {$[-0.61,0.16]$} & 1.35 & .246 \\
\hline $\begin{array}{l}\text { Tiempo de traslado casa-universidad-casa } \\
\text { (minutos/día) }\end{array}$ & 0.00 & 0.00 & {$[-0.01,0.01]$} & 0.85 & .356 \\
\hline Nivel socioeconómico ( 0 a 366 puntos) & 0.01 & 0.01 & {$[0.00,0.03]$} & 5.05 & .025 \\
\hline Escolaridad de la madre ( 0 a 72 puntos) & 0.01 & 0.02 & {$[-0.02,0.05]$} & 0.57 & .449 \\
\hline Escolaridad del padre ( 0 a 72 puntos) & -0.01 & 0.02 & {$[-0.04,0.02]$} & 0.73 & .394 \\
\hline (Escala) & 14.07 & 1.44 & {$[11.51,17.20]$} & & \\
\hline
\end{tabular}

Nota: $B=$ Coeficiente beta no estandarizado, $E E$ = Error Estándar, IC = Intervalo de Confianza. 
El análisis de mediante un modelo lineal generalizado para satisfacción con la vida (Tabla 3) mostró una asociación estadísticamente significativa de ésta con el nivel de actividad física practicado por los estudiantes, su sexo y su nivel socioeconómico, $\chi^{2}(8)=22.38, p$ $=.004$. Por otra parte, se encontró una asociación significativa de la resiliencia de los alumnos con el nivel de actividad física practicado, la educación del padre. Además, se observó una tendencia en la asociación entre la resiliencia de los estudiantes con su nivel socioeconómico y el tiempo que les tomaba diariamente trasladarse de la casa a la escuela y de regreso, $\chi^{2}(8)=27.18, p=.001$ (Tabla 4$)$.

Tabla 4

Modelo para predecir resiliencia

\begin{tabular}{|c|c|c|c|c|c|}
\hline Variable/Categoría & $\boldsymbol{B}$ & $\boldsymbol{E E}$ & IC 95\% & $\chi^{2}$ Wald & $p$ \\
\hline (Intercepto) & 23.13 & 5.59 & {$[12.18,34.08]$} & 17.14 & .000 \\
\hline $\begin{array}{l}\text { Nivel de actividad física practicado } \\
\text { Moderado/Alto } \\
\text { Bajo }\end{array}$ & $\begin{array}{c}0 \\
-2.41\end{array}$ & 0.74 & {$[-3.87,-0.95]$} & 10.51 & .001 \\
\hline $\begin{array}{l}\text { Sexo } \\
\quad \text { Mujer } \\
\text { Hombre }\end{array}$ & $\begin{array}{c}0 \\
0.08\end{array}$ & 0.67 & {$[-1.23,1.39]$} & 0.01 & .907 \\
\hline $\begin{array}{l}\text { Grado de estudio } \\
\text { Segundo } \\
\text { Primero }\end{array}$ & $\begin{array}{c}0 \\
1.01\end{array}$ & 0.66 & {$[-0.28,2.30]$} & 2.35 & .125 \\
\hline Edad (años) & -0.06 & 0.27 & {$[-0.59,0.48]$} & 0.04 & .836 \\
\hline $\begin{array}{l}\text { Tiempo de traslado casa-universidad-casa } \\
\text { (minutos/día) }\end{array}$ & -0.01 & 0.00 & {$[-0.02,0.00]$} & 2.81 & .094 \\
\hline Nivel socioeconómico ( 0 a 366 puntos) & 0.01 & 0.01 & {$[0.00,0.02]$} & 3.67 & .055 \\
\hline Escolaridad de la madre ( 0 a 72 puntos) & -0.02 & 0.02 & {$[-0.07,0.03]$} & 0.68 & .410 \\
\hline Escolaridad del padre ( 0 a 72 puntos) & -0.06 & 0.02 & {$[-0.10,-0.02]$} & 8.88 & .003 \\
\hline (Escala) & 17.83 & 1.83 & {$[14.58,21.80]$} & & \\
\hline
\end{tabular}

Nota: $B=$ Coeficiente beta no estandarizado, $E E=$ Error Estándar, IC = Intervalo de Confianza.

Con respecto al autoconcepto de los alumnos (Tabla 5), se observó una asociación significativa de éste con la educación del padre y el nivel socioeconómico; mientras que con el nivel de actividad física mostró una tendencia significativa, $\chi^{2}(8)=16.07, p=.041$.

Por último, no se encontró un modelo de asociación con significancia estadística entre el compromiso de los alumnos y las variables incluidas en el estudio, $\chi^{2}(8)=4.37, p=.822($ Tabla 6$)$.

\section{DISCUSIÓN}

El objetivo de esta investigación fue identificar la asociación entre los niveles de actividad física que practicaban los estudiantes de medicina de una universidad mexicana y aspectos positivos de su salud mental, considerando algunas variables sociodemográficas y el tiempo que les tomaba trasladarse de su casa a la escuela y de regreso. Los resultados de la presente investigación muestran que existe asociación entre la práctica de actividad física de los alumnos participantes, su satisfacción con la vida y su resiliencia.

\section{Actividad Física y Salud Mental Positiva}

Este análisis se realizó siguiendo las recomendaciones de otros investigadores (IPAQ, s.f.), en el sentido de optar por niveles de actividad física más elevados que permitieran observar una asociación más clara entre la actividad física practicada por los estudiantes y los beneficios en su salud mental. De este modo, fue posible identificar que quienes practicaban actividad física a un nivel bajo presentaban menor resiliencia y satisfacción con la vida que quienes la realizaban a un 


\section{Actividad física y salud mental en estudiantes de medicina mexicanos}

nivel moderado o alto. Estos resultados respaldan el hecho de que niveles altos de actividad física se asocian a mayor resiliencia, como se ha reportado en estudiantes universitarios (Chacón-Cuberos et al. 2017; Ozkara et al., 2016; Romero-Barquero, 2014) y trabajadores (Gómez-Chacón y Fernández-Martínez, 2020); así como aquellos estudios que encuentran esta misma relación con la satisfacción con la vida en diversas poblaciones como adolescentes (Reigal, Hernández-Mendo et al., 2020), estudiantes universitarios (Fernández-Ozcorta et al., 2015; Thome y Espelage, 2004) y residentes médicos (Lebensohn et al., 2013).

Tabla 5

Modelo para predecir autoconcepto

\begin{tabular}{|c|c|c|c|c|c|}
\hline Variable/Categoría & $\boldsymbol{B}$ & $\boldsymbol{E} \boldsymbol{E}$ & IC 95\% & $\chi^{2}$ Wald & $p$ \\
\hline (Intercepto) & 32.02 & 7.94 & {$[17.45,48.58]$} & 17.29 & .000 \\
\hline $\begin{array}{l}\text { Nivel de actividad física practicado } \\
\text { Moderado/Alto } \\
\text { Bajo }\end{array}$ & $\begin{array}{c}0 \\
-2.51\end{array}$ & 1.37 & {$[-5.21,0.18]$} & 3.33 & .068 \\
\hline $\begin{array}{l}\text { Sexo } \\
\quad \text { Mujer } \\
\text { Hombre }\end{array}$ & $\begin{array}{c}0 \\
-1.66\end{array}$ & 1.23 & {$[-4.06,0.75]$} & 1.83 & .176 \\
\hline $\begin{array}{l}\text { Grado de estudio } \\
\text { Segundo } \\
\text { Primero }\end{array}$ & $\begin{array}{c}0 \\
1.79\end{array}$ & 1.19 & {$[-0.53,4.12]$} & 2.28 & .131 \\
\hline Edad (años) & 0.29 & 0.37 & {$[-0.43,1.00]$} & 0.61 & .435 \\
\hline $\begin{array}{l}\text { Tiempo de traslado casa-universidad-casa } \\
\text { (minutos/día) }\end{array}$ & 0.01 & 0.01 & {$[-0.02,0.01]$} & 0.48 & .488 \\
\hline Nivel socioeconómico ( 0 a 366 puntos) & 0.03 & 0.01 & {$[0.00,0.05]$} & 4.83 & .028 \\
\hline Escolaridad de la madre ( 0 a 72 puntos) & -0.03 & 0.04 & {$[-0.10,0.04]$} & 0.72 & .397 \\
\hline Escolaridad del padre ( 0 a 72 puntos) & -0.09 & 0.04 & {$[-0.16,-0.02]$} & 5.57 & .018 \\
\hline (Escala) & 53.83 & 5.52 & [44.03, 65.82] & & \\
\hline
\end{tabular}

Nota: $B=$ Coeficiente beta no estandarizado, $E E=$ Error Estándar, IC = Intervalo de Confianza.

El autoconcepto mostró una tendencia de asociación estadística con la práctica de actividad física, lo cual no descarta del todo los hallazgos reportados sobre una asociación positiva en esta relación (Álvarez-Rivera, et al., 2015; Cachón-Zagalaz, et al., 2015; Dale et al., 2019; Kyle et al., 2016; Moral-Campillo, et al., 2020; Onetti-Onetti, et al., 2019). Sin embargo, el compromiso de los alumnos no mostró asociación alguna con la práctica de actividad física, lo cual fue contrario a lo señalado por Gómez-Chacón y Fernández-Martínez (2020). Una explicación propuesta en una revisión hecha por Wegner et al. (2014) podría brindar claridad con relación a este hallazgo; estos autores encontraron que la práctica de actividad física beneficiaba en mayor medida a poblaciones clínicas (grupos de personas que presentaban tasas más altas de depresión o ansiedad), por lo que no era de extrañar que los estudios con poblaciones no clínicas que buscaban dichas asociaciones (como el caso de esta investigación), en ocasiones, no encontraran correlaciones significativas.

\section{Sexo y Salud Mental Positiva}

En el caso de las variables sociodemográficas estudiadas, el hecho de ser mujer se asoció con tener mayor satisfacción con la vida; quizá porque, hasta cierto punto, las mujeres tienden a responder más asertivamente que los varones a situaciones o acciones que promuevan estilos de vida más saludables (Muller et al., 2006), lo que puede repercutir positivamente (en mayor medida que en el caso de los hombres), en algunos aspectos de su salud mental.

\section{Nivel Socioeconómico y Salud Mental Positiva}

Se encontró que mientras más elevado era el nivel socioeconómico de los estudiantes, mayor era su 


\section{Fouilloux, C., Fouilloux-Morales, M., Tafoya S.A., Petra-Micu, I.}

autoconcepto y su satisfacción con la vida. Esto último concuerda con los resultados obtenidos en un estudio llevado a cabo con alumnos noruegos, donde se observó que quienes tenían un mayor nivel

Tabla 6

Modelo para predecir compromiso

\begin{tabular}{|c|c|c|c|c|c|}
\hline Variable/Categoría & $\boldsymbol{B}$ & $E E$ & IC $95 \%$ & $\chi^{2}$ Wald & $p$ \\
\hline (Intercepto) & 59.77 & 11.10 & {$[38.00,81.53]$} & 28.97 & .000 \\
\hline $\begin{array}{l}\text { Nivel de actividad física practicado } \\
\text { Moderado/Alto } \\
\text { Bajo }\end{array}$ & $\begin{array}{c}0 \\
-1.89\end{array}$ & 1.94 & {$[-5.69,1.91]$} & 0.95 & .329 \\
\hline $\begin{array}{l}\text { Sexo } \\
\quad \text { Mujer } \\
\text { Hombre }\end{array}$ & $\begin{array}{c}0 \\
-0.93\end{array}$ & 1.74 & {$[-4.35,2.49]$} & 0.28 & .594 \\
\hline $\begin{array}{l}\text { Grado de estudio } \\
\text { Segundo } \\
\text { Primero }\end{array}$ & $\begin{array}{c}0 \\
0.19\end{array}$ & 1.89 & {$[-3.52,3.90]$} & 0.01 & .920 \\
\hline Edad (años) & 0.44 & 0.52 & {$[-0.57,1.45]$} & 0.72 & .398 \\
\hline $\begin{array}{l}\text { Tiempo de traslado casa-universidad-casa } \\
\text { (minutos/día) }\end{array}$ & 0.00 & 0.01 & {$[-0.03,0.02]$} & 0.03 & .872 \\
\hline Nivel socioeconómico (0 a 366 puntos) & 0.02 & 0.02 & {$[-0.02,0.05]$} & 1.05 & .306 \\
\hline Escolaridad de la madre ( 0 a 72 puntos) & 0.01 & 0.05 & {$[-0.10,0.11]$} & 0.01 & .922 \\
\hline Escolaridad del padre (0 a 72 puntos $)$ & -0.09 & 0.04 & {$[-0.18,-0.01]$} & 4.35 & .037 \\
\hline (Escala) & 116.06 & 11.91 & {$[94.92,141.92]$} & & \\
\hline
\end{tabular}

Nota: $B=$ Coeficiente beta no estandarizado, $E E$ = Error Estándar, IC = Intervalo de Confianza.

\section{Escolaridad del Padre y Salud Mental Positiva}

A diferencia de la escolaridad de la madre (donde no se encontró ningún tipo de asociación significativa), en el caso del padre, se observó que, en la medida en que el nivel educativo de éste aumentaba, los alumnos expresaban menor resiliencia y autoconcepto. No se encontraron estudios con respecto a la asociación entre la escolaridad del padre y aspectos positivos de la salud mental, por lo que se requieren investigaciones futuras que analicen dicha relación.

Excepto en el caso de la escolaridad de la madre, la mayoría de las variables sociodemográficas estudiadas (sexo, edad, grado escolar, nivel socioeconómico y educación del padre) presentaron una asociación significativa con algunos de los aspectos positivos de la salud mental (satisfacción con la vida, resiliencia, autoconcepto o compromiso). Desafortunadamente, debido a la naturaleza de estas variables sociodemográficas, resulta difícil, o incluso imposible, intervenir sobre ellas; por el contrario, la actividad socioeconómico presentaban una mayor satisfacción con la vida (Weinberg, Stevens, Duinhof y Finkenauer, 2019). 


\section{Actividad física y salud mental en estudiantes de medicina mexicanos}

que los alumnos tienen con su vida y su capacidad resiliente; lo que puede impactar de manera favorable en su desempeño académico (Zhou et al., 2014), así como facilitar la adaptación de quienes inician la carrera a sus altas exigencias, sin afectar negativamente su productividad académica $\mathrm{y}$ convivencia (Ríos-Bustos et al., 2017).

Asimismo, esta investigación sirve como referencia para futuros estudios que profundicen el análisis de los efectos positivos de la actividad física en la salud mental de otras poblaciones; exploren la inclusión de otros aspectos positivos en su análisis; y brinden mayor evidencia para sustentar la propuesta de implementar programas orientados a incrementar la práctica de actividad física en diferentes grupos, con el fin de promover su salud mental.

\section{APLICACIONES PRÁCTICAS}

Esta investigación proporciona información que puede ser útil para el diseño de un programa piloto de intervención que promueva la práctica de actividad física a niveles moderado y alto en estudiantes de medicina en México, lo que se espera favorezca el desarrollo de su resiliencia y satisfacción con la vida, con un impacto positivo en el ámbito académico y personal de sus vidas.

Será importante considerar al planear programas de intervención que (si bien éstos deben estar orientados a toda la población estudiantil) los alumnos varones o con un nivel socioeconómico bajo, requieren de mayor atención para motivarlos a practicar actividad física, con la intención de promover el desarrollo de aspectos positivos de su salud mental.

Por último, aunque la información anterior puede ser útil como punto de partida para intervenciones con poblaciones similares a la de esta investigación; será necesario que, en futuros estudios, se profundice sobre la asociación de la salud mental positiva, no sólo con la actividad física, sino también con otras variables como las sociodemográficas analizadas en el presente trabajo.

\section{CONFLICTO DE INTERESES}

Los autores declaran no tener conflicto de interés.

\section{REFERENCIAS}

1. Almagro-Valverde, S., Dueñas-Guzmán, M.A., Tercedor-Sánchez, P. (2014). Actividad física y depresión: revisión sistemática. Revista Internacional de Medicina y Ciencias de la Actividad Física y el Deporte, 14(54), 377-392. Disponible en: http://cdeporte.rediris.es/revista/revista54/artactiv idad472.pdf

2. Álvarez-Rivera, L., Cuevas-Ferrera, R., Lara-Pot, A., González-Hernández, J. (2015). Diferencias del autoconcepto físico en practicantes y no practicantes de actividad física en estudiantes universitarios. Cuadernos de Psicología del Deporte, 15(2), https://doi.org/10.4321/S157884232015000200004

3. Atienza, F.L., Pons, D., Balaguer, I., García-Merita, M. (2000). Propiedades psicométricas de la Escala de Satisfacción con la Vida en Adolescentes. Psicothema, 12(2), 314-319. Disponible en: https://www.redalyc.org/articulo.oa?id=727/7271 2226

4. Babenko, O., Mosewich, A. (2017). In sport and now in medical school: examining students' wellbeing and motivations for learning. International Journal of Medical Education, 8, 336-342. https://doi.org/10.5116/ijme.59b7.8023

5. Babenko, O., Mosewich, A., Abraham, J., Lai, H. (2018). Contributions of psychological needs, self-compassion, leisure-time exercise, and achievement goals to academic engagement and exhaustion in Canadian medical students. Journal of Educational Evaluation for Health Professions, 15, 2. https://doi.org/10.3352/jeehp.2018.15.2

6. Bass, M.A., Enochs, W.K., DiBrezzo, R. (2002). Comparison of two exercise programs on general well-being of college students. Psychological Reports, $91(3 \quad \mathrm{Pt} \quad 2), \quad 1195-1201$. https://doi.org/10.2466/pr0.2002.91.3f.1195

7. Borbón-Castro, N.A., Castro-Zamora, A.A., CruzCastruita, R.M., Banda-Sauceda, N.C., de la Cruz- 


\section{Fouilloux, C., Fouilloux-Morales, M., Tafoya S.A., Petra-Micu, I.}

Ortega, M.F. (2020). The effects of a multidimensional exercise program on health behavior and biopsychological factors in Mexican older adults. Frontiers in Psychology, 10. https://doi.org/10.3389/fpsyg.2019.02668

8. Cachón-Zagalaz, J., Cuervo-Tuero, C., ZagalazSánchez, M.L., González-González de Mesa, C. (2015). Relación entre la práctica deportiva y las dimensiones del autoconcepto en función del género y la especialidad que cursan los estudiantes del grado de magisterio. Journal of Sport and Health Research, 7(3), 257-266.

9. Caspersen, C.J., Powell, K.E., Christenson, G.M. (1985). Physical activity, exercise, and physical fitness: definitions and distinctions for healthrelated research. Public Health Reports, 100(2), 126-131.

10. Chacón-Cuberos, R, Puertas-Molero, P., PérezCortés, A.J. (2017). Niveles de resiliencia según práctica de actividad física en estudiantes universitarios de Educación Física. Education, Sport, Health and Physical Activity, 1(1), 59-67.

11. Craig, C.L., Marshall, A.L., Sjöström, M., Bauman, A.E., Booth, M.L., Ainsworth, B.E., ... Oja, P. (2003). International Physical Activity Questionnaire: 12-country reliability and validity. Medicine and Science in Sports and Exercise, 35(8), 1381-1395. https://doi.org/10.1249/01.MSS.0000078924.614 53.FB

12. Dale, L.P., Vanderloo, L., Moore, S., Faulkner, G. (2019). Physical activity and depression, anxiety, and self-esteem in children and youth: an umbrella systematic review. Mental Health and Physical Activity, 16, 66-79. https://doi.org/10.1016/j.mhpa.2018.12.001

13. Diener, E., Emmons, R.A., Larsen, R.J., Griffin, S. (1985). The satisfaction with life scale. Journal of Personality Assessment, 49(1), 71-75. https://doi.org/10.1207/s15327752jpa4901_13

14. Fernández-Ozcorta, E.J., Almagro-Torres, B.J., Sáenz-López-Buñuel, P. (2015). Inteligencia emocional percibida y el bienestar psicológico de estudiantes universitarios en función del nivel de actividad física. Cultura Ciencia Deporte (CCD), 10(28), 31-39. https://doi.org/10.12800/ccd.v10i28.513

15. Fox, J. (2015). Generalized Linear Models. En: Applied regression analysis and generalized linear models (3a ed.). Los Angeles: Sage Publications.

16. Galván-Molina, J.F., Jiménez-Capdeville, M.E., Hernández-Mata, J.M., Arellano-Cano, J.R. (2017). Psychopathology screening in medical school students. Gaceta Médica de México, 153, 69-80. Disponible en: https://www.anmm.org.mx/GMM/2017/n1_engli sh/3942AX171_153_2017_UK1_069-080.pdf

17. Gómez-Chacón, R., Fernández-Martínez, N. (2020). Relación entre la práctica de actividad física y los empleados saludables en un club deportivo-social. Cuadernos de Psicología del Deporte, 20(3), 65-73. Disponible en: https://revistas.um.es/cpd/article/view/389761/28 5551

18. International Physical Activity Questionnaire (s.f.). IPAQ: International Physical Activity Questionnaire. Disponible en: https://sites.google.com/site/theipaq/

19. Jahoda, M. (1958). Current concepts of positive mental health. Nuevo Hampshire: Ayer Company Publishers.

20. Jamali, A., Tofangchiha, S., Jamali, R., Nedjat, S., Jan, D., Narimani, A., Montazeri, A. (2013). Medical students' health-related quality of life: roles of social and behavioural factors. Medical Education, $\quad 47(10), \quad$ 1001-1012. https://doi.org/10.1111/medu.12247

21. Kyle, T.L., Hernández-Mendo, A., ReigalGarrido, R.E., Morales-Sánchez, V. (2016). Efectos de la actividad física en el autoconcepto y la autoeficacia en preadolescentes. Retos, 29, 6165.

22. Lebensohn, P., Dodds, S., Benn, R., Brooks, A.J., Birch, M., Cook, P., ... Maizes, V. (2013). 


\section{Actividad física y salud mental en estudiantes de medicina mexicanos}

Resident wellness behaviors: relationship to stress, depression, and burnout. Family Medicine, 45(8), 541-549. Disponible en: https://fammedarchives.blob.core.windows.net/i magesandpdfs/fmhub/fm2013/September/Patricia 541.pdf

23. Lee, E., Spence, J.C., Tremblay, M.S., Carson, V. (2018). Meeting 24-Hour Movement Guidelines for Children and Youth and associations with psychological well-being among South Korean adolescents. Mental Health and Physical Activity, 14 , 66-73. https://doi.org/10.1016/j.mhpa.2018.02.001

24. Ligeza, T.S, Kałamała, P., Tarnawczyk, O., Maciejczyk, M., Wyczesany, M. (2019). Frequent physical exercise is associated with better ability to regulate negative emotions in adult women: the electrophysiological evidence. Mental Health and Physical Activity, 17, 100294. https://doi.org/10.1016/j.mhpa.2019.100294

25. López-Bárcena, J.J., González de Cossio-Ortiz, M.G., Rodríguez-Gutiérrez, M.C. (2006). Actividad física en estudiantes universitarios: prevalencia, características y tendencia. Medicina Interna de México, 22(3), 189-196. Disponible en: https://www.cmim.org/Revista/2006/200603_ma $y_{\_}$jun.pdf\#page $=31$

26. López-Walle, J., Tristán, J., Tomás, I., GallegosGuajardo, J., Góngora, E., Hernández-Pozo, M.R. (2020). Estrés percibido y felicidad auténtica a través del nivel de actividad física en jóvenes universitarios. Cuadernos de Psicología del Deporte, 20(2), 265-275. https://doi.org/10.6018/cpd.358601

27. Loue, S. (2006). Aspectos éticos de los estudios multicéntricos. En F. Lolas, A. Quezada, E. Rodríguez (Eds.), Investigación en salud: dimensión ética, 273-278. Chile: CIEB, Universidad de Chile.

28. Medina, M.E., Borges, G., Lara, C., Benjet, C., Blanco, J., Fleiz, C. ... Aguilar, S. (2003). Prevalencia de trastornos mentales y uso de servicios: resultados de la Encuesta Nacional de
Epidemiología Psiquiátrica en México. Salud Mental, 26(4), 1-16.

29. Moral-Campillo, L., Reigal-Garrido, R.E., Hernández-Mendo, A. (2020). Actividad física, funcionamiento cognitivo y psicosocial en una muestra preadolescente. Revista de Psicología del Deporte, 29(1). 123-132. Disponible en: https://ddd.uab.cat/record/216427

30. Morales, P. (2006). Medición de actitudes en psicología y educación: construcción de escalas y problemas metodológicos (3a ed.). Madrid: Universidad Pontificia Comillas.

31. Muller, S.M., Dennis, D.L., Gorrow, T. (2006). Emotional well-being of college students in health courses with and without an exercise component. Perceptual and Motor Skills, 103(3), 717-725. https://doi.org/10.2466\%2Fpms.103.3.717-725

32. Nogueira-López, A., Salguero del Valle, A., Márquez-Rosa, S. (2017). Adicción a correr: una revisión desde sus inicios hasta la actualidad. Revista de Psicología Aplicada al Deporte y al Ejercicio Físico, 1(e6), 1-22. https://doi.org/10.5093/rpadef2017a1

33. Onetti-Onetti, W., Chinchilla-Minguet, J.L., Lourenço Martins, F.M., Castillo-Rodríguez, A. (2019). Self-concept and physical activity: differences between high school and university students in Spain and Portugal. Frontiers in Psychology, 10, 1313. https://doi.org/10.3389/fpsyg.2019.01333

34. Organización Mundial de la Salud (2001). Informe sobre la salud en el mundo 2001 - Salud mental: nuevos conocimientos, nuevas esperanzas. Ginebra: OMS. Disponible en: https://www.who.int/whr/2001/es/

35. Organización Mundial de la Salud. (2005). Promoting mental health: concepts, emerging evidence, practice. A report of the World Health Organization, Department of Mental Health and Substance Abuse in collaboration with the Victorian Health Promotion Foundation and the University of Melbourne. World Health 


\section{Fouilloux, C., Fouilloux-Morales, M., Tafoya S.A., Petra-Micu, I.}

Organization.

https://apps.who.int/iris/handle/10665/43286.

36. Organización Mundial de la Salud (s.f.). Estrategia mundial sobre régimen alimentario, actividad física $y$ salud. Disponible en: https://www.who.int/dietphysicalactivity/pa/es/

37. Ortiz-León, S., Arechavaleta-Hernández, B., Tafoya-Ramos, S.A., Fouilloux-Morales, C. (2007). Riesgo de enfermedad mental en internos de pregrado de la Facultad de Medicina de la UNAM. Psiquiatría, 23, 1-3.

38. Ozkara, A.B., Kalkavan, A., Alemdag, S., Alemdag, C. (2016). The role of physical activity in psychological resilience. Baltic Journal of Sport and Health Sciences, 3(102), 24-29. https://doi.org/10.33607/bjshs.v3i102.62

39. Peralta-Díaz, S.C., Ramírez-Giraldo, A.F., Castaño-Buitrago, H. (2006). Factores resilientes asociados al rendimiento académico en estudiantes pertenecientes a la Universidad de Sucre (Colombia). Psicología desde el Caribe, 17, 196-219. Disponible en: http://rcientificas.uninorte.edu.co/index.php/psico logia/article/view/2054

40. Reigal, R.E., Hernández-Mendo, A., Juárez-Ruiz de Mier, R., Morales-Sánchez, V. (2020). Physical exercise and fitness level are related to cognitive and psychosocial functioning in adolescents. Frontiers in Psychology, 11, 1777. https://doi.org/ 10.3389/fpsyg.2020.01777

41. Reigal, R.E., Moral-Campillo, L., Morillo-Baro, J.P., Juárez-Ruiz de Mier, R., Hernández-Mendo, A., Morales-Sánchez, V. (2020). Physical exercise, fitness, cognitive functioning, and psychosocial variables in an adolescent sample. International Journal of Environmental Research and Public Health, 17(3), 1100. https://doi.org/10.3390/ijerph17031100

42. Reigal, R., Videra, A., Gil, J. (2014). Physical exercise, general self-efficacy and life satisfaction in adolescence. International Journal of Medicine and Science of Physical Activity and Sport, 14(55), 561-576. Disponible en: http://cdeporte.rediris.es/revista/revista55/artdise no480.htm

43. Ríos-Bustos, M.E., Torres-Vaca, M., de la Torre, C. (2017). Actividad física y adaptación escolar en estudiantes de medicina en un campus de la Universidad Nacional Autónoma de México. Investigación en Educación Médica, 6(21), 16-24. http://doi.org/10.1016/j.riem.2016.04.003

44. Rodríguez-Romo, G., Barriopedro, M., AlonsoSalazar, P.J., Garrido-Muñoz, M. (2015). Relaciones entre actividad física y salud mental en la población adulta de Madrid. Revista de Psicología del Deporte, 24(2), 233-239. Disponible en: http://www.redalyc.org/articulo.oa?id=23514141 3005

45. Romero-Barquero, C.E. (2014). Relación entre resiliencia, práctica de actividades recreativas físicas y consumo de alcohol en colegiales. EmásF: Revista Digital de Educación Física, 26, 59-72. Disponible en: https://dialnet.unirioja.es/servlet/articulo?codigo= 4723948

46. Sánchez-Beleña, F., Alejo García-Naveira, V. (2017). Sobreentrenamiento y deporte desde una perspectiva psicológica: estado de la cuestión. Revista de Psicología Aplicada al Deporte y al Ejercicio Físico, 2(e12), 1-12. https://doi.org/10.5093/rpadef2017a8

47. Schaufeli, W.B., Salanova, M., González, V., Bakker, A.B. (2002). The measurement of engagement and burnout: a two-sample confirmatory factor analytic approach. Journal of Happiness Studies, 3(1), 71-92. https://doi.org/10.1023/A:1015630930326

48. Smith, B.W., Dalen, J., Wiggins, K., Tooley, E., Christopher, P., Bernard, J. (2008). The brief resilience scale: assessing the ability to bounce back. International Journal of Behavioral Medicine, 15(3), 194-200. https://doi.org/10.1080/10705500802222972

49. Tamminen, N., Reinikainen, J., AppelqvistSchmidlechner, K., Borodulin, K., Mäki-Opas, T., 


\section{Actividad física y salud mental en estudiantes de medicina mexicanos}

Solin, P. (2020). Associations of physical activity with positive mental health: A populationbased study. Mental Health and Physical Activity, 18, 100319.

https://doi.org/10.1016/j.mhpa.2020.100319

50. Terebessy, A., Czeglédi, E., Balla, B.C., Horváth, F., Balázs, P. (2016). Medical students' health behaviour and self-reported mental health status by their country of origin: a cross-sectional study. BMC Psychiatry, $16, \quad 171$. http://doi.org/10.1186/s12888-016-0884-8

51. Thome, J., Espelage, D.L. (2004). Relations among exercise, coping, disordered eating, and psychological health among college students. Eating Behaviors, 5(4), 337-351. https://doi.org/10.1016/j.eatbeh.2004.04.002

52. Wegner, M., Helmich, I., Machado, S., Nardi, A.E., Arias-Carrión, O., Budde, H. (2014). Effects of exercise on anxiety and depression disorders: review of meta-analyses and neurobiological mechanisms. CNS \& Neurological Disorders Drug Targets, 13, 1002-1014. https://doi.org/10.2174/187152731366614061210 2841

53. Weinberg, D., Stevens, G.W.J.M., Duinhof, E.L., Finkenauer, C. (2019). Adolescent socioeconomic status and mental health inequalities in the Netherlands, 2001-2017. International Journal of Environmental Research Public Health, 16, 3605. https://doi.org/10.3390/ijerph16193605

54. Zhou, YX, Zhao, Z.T., Li, L., Wan, C.S., Peng, C.H., Yang, J., Ou, C.Q. (2014). Predictors of first-year GPA of medical students: longitudinal study of 1285 matriculates in China. BMC Medical Education, 14, 87. http://doi.org/10.1186/1472-6920-14-87 